\title{
Simulation of Optical Coherence Elastography in Agar Based on Finite Element Analysis
}

\author{
Xingming Tao ${ }^{1}$, Lihua Fang ${ }^{1, *}$, Luchao $\operatorname{Lin}^{1}$, Ruirui $\mathrm{Du}^{1}$, Yinyu Song ${ }^{1}$ \\ ${ }^{1}$ Key Laboratory of Nondestructive Test (Ministry of Education), Nanchang Hangkong University, Nanchang, 330063, China
}

\begin{abstract}
The finite element method is used to simulate the optical coherent elastic imaging in Agar. The shear wave velocity in Agar was measured by ARF-OCE system, and then the Agar model was established by finite element method, and then the shear wave velocity in Agar model was measured. The shear wave velocity in experiment and finite element simulation were compared and analyzed. The shear wave velocity obtained in the experiment is $2.50 \mathrm{~m} / \mathrm{s}$, and the range of shear wave velocity obtained in the finite element simulation is $2.4802 \mathrm{~m} / \mathrm{s}$, and the average wave velocity is $2.5167 \mathrm{~m} / \mathrm{s}$. The finite element method can express tissue elasticity directly and clearly, and it plays a great guiding role in corneal elastography.
\end{abstract}

\section{Introduction}

The mechanical properties of tissue (such as elasticity) have important clinical significance for the detection and diagnosis of various diseases [1]. Evaluating the propagation velocity of elastic waves in tissue is an important method for quantitative measurement of tissue elasticity, which has been widely used in ultrasonic imaging [2], magnetic resonance imaging [3], optical technology [4] and other imaging methods. Therefore, visualizing the propagation of elastic waves in tissues at ultra-high frame frequency directly, it can provide more clearer information about tissue biomechanical properties. This is beneficial to the rapid detection of clinical tissue and high-resolution tissue elastography.

Since Ophir put forward the concept of elastography in 1991 [5], it has been concerned by the medical profession and developed rapidly. The basic principle of elastic imaging is to provide internal or external, dynamic or static excitation on the biological tissue. Under the action of the laws of physics, the tissue will produce responses such as displacement, strain and velocity, and their distribution will change with the excitation [6]. These changes can be transformed into images, and the elastic distribution of mechanical properties within the tissue can be obtained. The current ultra-fast ultrasonic imaging technology, can display the propagation of shear waves in tissue at a frame rate of dozens of $\mathrm{kHz}$ [2].Elastography images (2D and 3D) can be generated according to wave velocity and have been successfully applied to clinical detection, such as thyroid [7], breast [8] ,liver [9] and so on. However, the relatively low depth resolution of ultrasonic imaging may hinder its application in the detection of small targets. This limitation becomes more pronounced when dealing

\footnotetext{
* Corresponding author: fanglh71@126.com
}

with eye tissue, such as the cornea, because the axial sampling points are greatly reduced as the sample thickness decreases (less than $1 \mathrm{~mm}$ ) [10]. In addition, in order to detect effectively, this low spatial resolution requires relatively high tissue deformation (tens of microns), which may cause damage to fragile soft tissue (such as cornea). At present, the latest measurement technology of elastic modulus of eye tissue [11] is acoustic radiation force optical coherence elastography (ARF-OCE). This technology has the advantages of high scanning speed, high resolution, and can realize elastic and structural imaging at the same time, so it will become a new generation of non-invasive tissue elastography mode [12].

Finite element method is an important method to study the mechanism of human tissue [13]. It can simulate the strain distribution of biological soft tissue and study the factors that can affect elastic imaging, such as different mechanical and geometric parameters of soft tissue. Elastography of tissue in vitro is the basis for the study of elastography. With the development of instrument technology, the measurement of clinical data is becoming more and more accurate, so the accurate construction of finite element model has become a reality. The finite element analysis method has strong modeling ability and high calculation accuracy. It can quantitatively analyze the deformation of tissue, display the distribution of stress and strain in tissue directly, and simulate the propagation of shear wave and surface Rayleigh wave. It is a powerful simulation tool for mechanism of optical coherence elastography(OCE) [14].

The aims of this paper is to verify the finite element method, and study its role in optical coherence elastography by comparing the shear wave velocity of Agar in experiment and finite element simulation. The purpose of this paper is to provide technical support for studying the mechanism of corneal elastography and 
improving the accuracy of elastography.

\section{Materials and methods}

\subsection{Experimental measurement}

The ARF-OCE system is mainly composed of ultrasonic transducer and OCT system. The overall scheme of the experimental device is shown in fig.1. In order to deform the Agar and generate elastic waves in it, we use an ultrasonic transducer with a central frequency of $4.5 \mathrm{MHz}$ to generate acoustic radiation force pressure pulses.

In order to measure the propagation velocity of elastic waves in Agar, we use a laser with light source wavelength $1310 \mathrm{~nm}$. The stability of the system is measured by experiments, and the axial resolution of $10.1 \mu \mathrm{m}$ can be achieved. Triggers the OCT system to synchronize with the function generator. The transverse scanning is carried out along the propagation direction of the wave, the scanning distance is $4 \mathrm{~mm}$, the scanning points are 2000, each point is scanned 500 times, the time is $10 \mathrm{~ms}$, and the sampling frequency is $50 \mathrm{KHz}$.

After measuring the time dependence of the displacement of each point, the time delay of the elastic wave is calculated by using the method of maximizing the cross-correlation function between the initial point and the measured point. Then, in the process of wave propagation, the relationship between the wave delay and the distance to the source is approximated as a linear function, and the wave velocity is calculated as the reciprocal of the corresponding linear slope. Using the known relation of shear wave velocity in incompressible medium as shown in formula (1), the obtained shear wave velocity is converted into Young's modulus.

$$
E=(1+v) \rho \mathcal{C}_{\mathrm{s}}^{2}
$$

The density $\rho$ and Poisson's ratio $v$ are assumed to be $1000 \mathrm{~kg} / \mathrm{m}^{3}$ and 0.5 respectively.

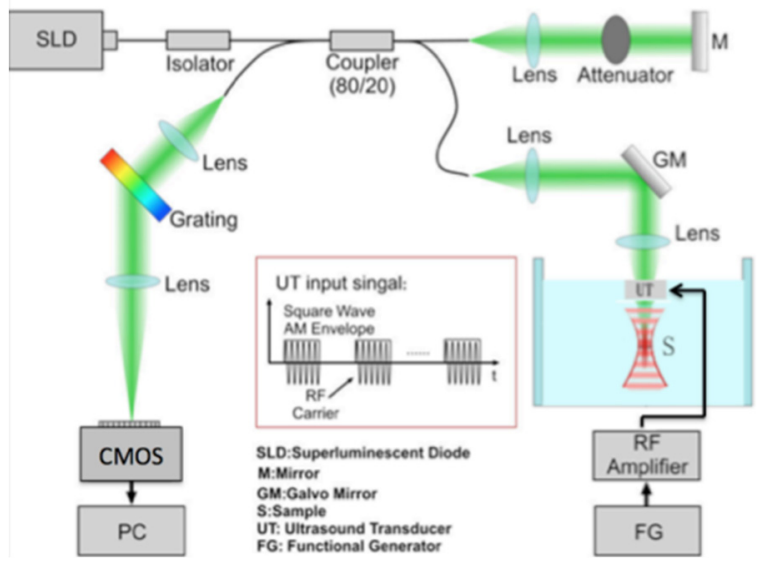

Fig. 1. The overall scheme of the experimental device

\subsection{Finite element simulation}

The finite element simulation flow chart is shown in fig.2. Firstly, a three-dimensional Agar model was established by using COMSOL Multiphysics software. Then the free tetrahedral element mesh type is used to divide the Agar model, and the mesh density meets the accuracy of the calculation. Then, according to the experimental scheme, Agar was made of almost incompressible, linear and uniform material. The density $\rho$ of Agar was set to $1000 \mathrm{~kg} / \mathrm{m}^{3}$, Poisson's ratio $v$ was set to 0.499 , and Young's modulus $E$ was set to $18.76 \mathrm{kPa}$. Finally, the transient dynamic analysis was used, and then, the zero displacement constraint is applied to the bottom of the Agar (in order to prevent the rotation of the Agar), and then the nodes are selected in the Agar model to apply pulse excitation.
According to the propagation of the shear wave in the sample, a number of observation points are set on the path of the shear wave propagation direction, and the displacement time data of each observation point are extracted, and then these data are drawn into a time-displacement graph by the analysis software. According to the displacement peak line in the time-displacement diagram, a straight line is synthesized and its slope is obtained through the algorithm, and the velocity is calculated by the formula (2) $\Delta v=\frac{\Delta x}{\Delta t}$. Where $\Delta v$ "is the velocity, $\Delta x "$ is the selected displacement, and $\Delta t$ "is the selected time. The calculated velocity is the shear wave velocity $c_{s}$ propagating in the sample. 


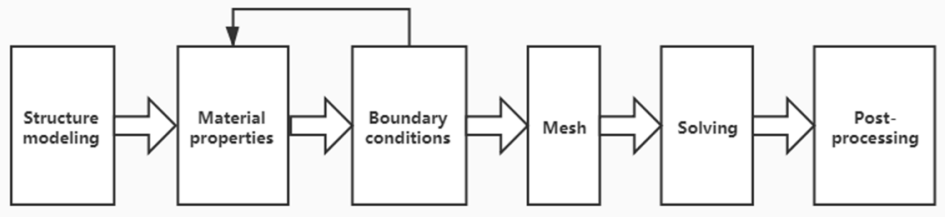

Fig. 2. Flow chart of finite element simulation

\section{Result}

\subsection{Shear wave velocity in experiment}

Fig.3 shows the propagation velocity of shear waves in Agar measured by ARF-OCE system. In $0.5 \%$ uniform Agar, the wave velocity measured by the system is $2.50 \mathrm{~m} / \mathrm{s}$. The Young's modulus $E=18.76 \mathrm{KPa}$ can be calculated from formula (1).

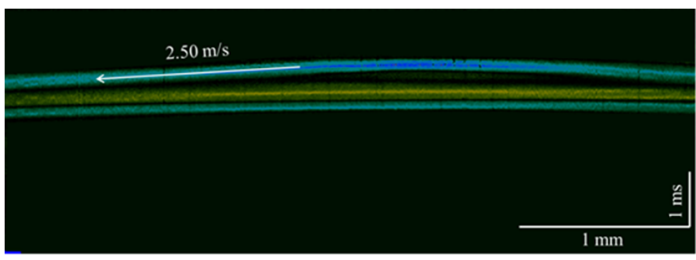

Fig. 3. Shear wave velocity of Agar in experiment

\subsection{Shear wave velocity in finite element simulation}

Fig.4 shows the time-displacement diagram of shear wave propagation in Agar measured by finite element method. Fig.5 shows the slope of the peak displacement line fitted by the algorithm.

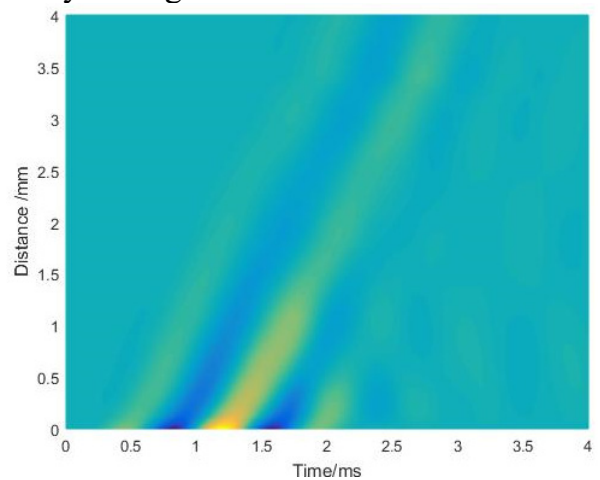

Fig. 4. Time-displacement diagram

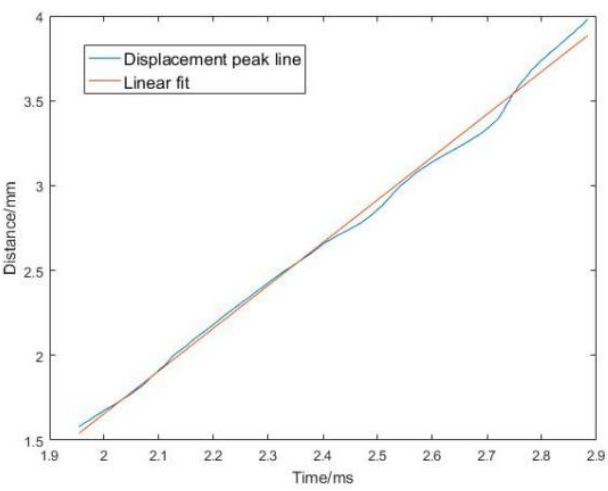

Fig. 5. Slope diagram
As can be seen from fig.5, the slope of the fitted straight line is $K=2.5189$, that is, the shear wave velocity $c_{s}=2.5189 \mathrm{~m} / \mathrm{s}$. We have done a total of four groups of simulation experiments, this is the simulation results of one group. According to the finite element method, we get that the range of shear wave velocity is $2.4802 \mathrm{~m} / \mathrm{s} \sim 2.5358 \mathrm{~m} / \mathrm{s}$, and the average velocity is $2.5167 \mathrm{~m} / \mathrm{s}$.

\subsection{Comparative Analysis of experiment and finite element Simulation}

From the results in sections 3.1 and 3.2 , it can be seen that the error range between the finite element simulation results and the experimental results is within $1.432 \%$, while the error between the average wave velocity in the simulation and the experimental wave velocity is $0.668 \%$.

\section{Conclusion}

In this paper, a finite element method is proposed to simulate optical coherence elastography in Agar. The results show that by comparing the shear wave velocity in the simulation results with the experimental results, the finite element method can express the tissue elasticity directly and clearly, which makes it play a great guiding role in corneal elastography.

\section{References}

1. Shah, S., M. Laiquzzaman, R. Bhojwani, S. Mantry, and I. Cunliffe, Assessment of the biomechanical properties of the cornea with the ocular response analyzer in normal and keratoconic eyes. Invest Ophth Vis Sci, 48(7): p. 3026-3031 (2007)

2. Bercoff, J., M. Tanter, S. Chaffai, and M. Fink, Ultrafast imaging of beamformed shear waves induced by the acoustic radiation force. Application to transient elas. IEEE, 2: p. 1899-1902 (2002)

3. Manduca, A., R. Muthupillai, P.J. Rossman, J.F. Greenleaf, and R.L. Ehman, Visualization of tissue elasticity by magnetic resonance elastography. Springer, Berlin, Heidelberg,P. 63-68 (1996)

4. Li, C., G. Guan, X. Cheng, Z. Huang, and R.K. Wang, Quantitative elastography provided by surface acoustic waves measured by phase-sensitive optical coherence tomography. Opt Lett, 37(4): p. 722-724 (2012)

5. Ophir, J., A Quantitative Method for Imaging the 
Elasticity of Biological Tissue. Ultrason Imag, 13: p. 111-134 (1991)

6. P. Wang,F. and Yang.Advances in New Technologies for Tissue Elastography[J].Laser Biol,25(6): 501-508,514(2016)(in Chinese).

7. Gangadhar, K., D.S. Hippe, J. Thiel, and M. Dighe, Impact of Image Orientation on Measurements of Thyroid Nodule Stiffness Using Shear Wave Elastography. J Ultras Med, 35(8): p. 1661 (2016)

8. Youk, J., H.M. Gweon, E.J. Son, J. Chung, J.A. Kim, and E.K. Kim, Three-dimensional shear-wave elastography for differentiating benign and malignant breast lesions: comparison with two-dimensional shear-wave elastography. Eur Radiol, 23(6): P. 1519-1527 (2013)

9. Cassinotto, C., B. Lapuyade, A. Mouries, J.B. Hiriart, J. Vergniol, D. Gaye, C. Castain, B.L. Bail, F. Chermak, and J. Foucher, Non-invasive assessment of liver fibrosis with impulse elastography: Comparison of Supersonic Shear Imaging with ARFI and FibroScan. J Hepatol, 61(3): p. 550-557 (2014)
10. Tanter, M., D. Touboul, J.L. Gennisson, J. Bercoff, and M. Fink, High-Resolution Quantitative Imaging of Cornea Elasticity Using Supersonic Shear Imaging. Medical Imaging, IEEE , 28(12): p.1881-1893 (2009)

11. Zhu, J., Y. Qu, T. Ma, R. Li, Y. Du, S. Huang, K.K. Shung, Q. Zhou, and Z. Chen, Imaging and characterizing shear wave and shear modulus under orthogonal acoustic radiation force excitation using OCT Doppler variance method. Opt Lett, 40(9): P. 2099-2102 (2015)

12. Qu, Y., M. Teng, Y. He, Z. Jiang, and Z. Chen, Acoustic Radiation Force Optical Coherence Elastography of Corneal Tissue. IEEE J Sel Top Quant, 22(3): p. 1-1 (2016)

13. J. Luo,J. Bai.Finite Element Analysis in Simelations of Ultrasound Elastography[J]. Beijing Biomed Eng,22(2):99-103 (2003)(in Chinese).

14. Han, Z., J. Li, M. Singh, S. Vantipalli, and K.V. Larin. Assessing the viscoelasticity of green light induced $C X L$ in the rabbit cornea by noncontact OCE and FEM. in SPIE BiOS.9693: P. 96930X (2016) 